\title{
Spline Computation for Solving Magnetohidrodynamics Free Convection Flow \\ ${ }^{1}$ Faraidun K. Hamasalh, ${ }^{2}$ Joseph G. and ${ }^{3}$ Najmadeen G. \\ ${ }^{1}$ Department of Mathematics,Collegeof Science Education, University of Sulaimani faraidunsalh@gmail.com \\ ${ }^{2}$ Faculty of Science, University of Zakho, Kurdistan -Iraq. \\ Joseph1955@yahoo.com \\ ${ }^{3}$ College of Science Education, University of Sulaimani, Kurdistan-Iraq. \\ Najm1961@gmail.com
}

\section{ABSTRACT}

In this paper, we construct numerical algorithms for solving Magnetohidrodynamics(MHD) free convection flow rate which has been discussed in detail. It is observed that, for a nonlinear system of differential equation, the spline model of nine degree is used, effectiveness and accuracy of this new method are presented. Several theorems relating the order of the ninth spline to the saturation of the estimator are proved. Some results of consistency are given and an application MHD system is given.

AMS SUBJECT CLASSIFICATION: 41A25, 65H10, 47E05.

KEYWORD:Splineinterpolation, Convergence analysis, MHD convection flow,System of nonlinear ordinary differential equations.

\section{Council for Innovative Research}

Peer Review Research Publishing System

\section{Journal: INTERNATIONAL JOURNAL OF COMPUTERS \& TECHNOLOGY}

\author{
Vol 5, No 3 \\ editor@cirworld.com \\ www.cirworld.com, member.cirworld.com
}




\section{INTRODUCTION}

Consider the system of nonlinear equations

$\frac{d A}{d t}=f\left(t, u_{1}, u_{2}, u_{3}, \ldots, u_{n}\right)$

$A\left(t_{0}\right)=\eta$

where $\mathrm{A}=\left[u_{1}, u_{2}, u_{3}, \ldots, u_{n}\right]^{T}, f=\left[f_{1}, f_{2}, f_{3}, \ldots ., f_{n}\right]^{T}, \eta=\left[\eta_{1}, \eta_{2}, \eta_{3}, \ldots, \eta_{n}\right]$.

Many problems in applied sciences and engineering are modeled as system of differential equations such as spring-mass systems, bending of beams, Magnetohidrodynamics free convection flow (MHD), chemical reactions and so forth can be formulated in terms of differential equations. Since the system of differential equations has wide applications in scientific research, so we consider the convective flow in fluid saturated porous medium which has been the subject of several recent papers. Therefore faster and accurate numerical solutions to this problem is very important, see([1-3], [5], and [12]).

There are several methods that can be used to solve the nonlinear problems numerically. A broad class of analytical solutions methods, such as Runge-Kutta of order six, Taylors series, Hirota's bilinear scheme and Hereman's method as [6-8], [10] and[12], were used to handle these problems. However, some of spline approximation had been proposed by ([2], [4] and [11]]) solved the system of differential equations and some initial value problems.

In the present paper, we discussed the convergence analysis of the ninth spline method for system of differential equations with new constraint and boundary conditions, also we apply this new model for solving magnetohidrodynamics free convection flow and mass transfer over a stretching sheet which has been analyzed numerically including the dufour and sort effects. We will use the function, two, fourth and seventh boundary conditions, to constructed the ninth spline with two initial conditions.

\section{DESCRIPTION OF THE METHOD}

We present a ninth spline interpolation for one dimensional and for a given sufficiently smooth function $f(x)$ defined on the interval $\mathrm{I}=[\mathrm{a}, \mathrm{b}]$, and $\Delta_{n}: a=x_{0}<x_{1}<x_{2}<\ldots<x_{n}=b$, denote the uniform partition of $I$ with knots $x_{i}=a+i h$, where $i=0,1,2, \ldots, n-1$ and $h=\frac{b-a}{n}$ is the length of each subintervals, and $\mathrm{d}$ the ninth spline is denoted by $S_{\Delta}(x)$ and defined on I as:

$s_{0}(x)=y_{0}+\left(x-x_{0}\right) y_{0}^{\prime}+\frac{\left(x-x_{0}\right)^{2}}{2} y_{0}^{\prime \prime}+\frac{\left(x-x_{0}\right)^{3}}{6} y_{0}^{\prime \prime \prime}+\frac{\left(x-x_{0}\right)^{4}}{24} y_{0}^{(4)}+\frac{\left(x-x_{0}\right)^{5}}{120} a_{0,5}+$ $\left(x-x_{0}\right)^{6} a_{0,6}+\frac{\left(x-x_{0}\right)^{7}}{5040} y_{0}^{(7)}+\left(x-x_{0}\right)^{8} a_{0,8}+\left(x-x_{0}\right)^{9} a_{0,9}(2)$

on the subinterval $\left[x_{0}, x_{1}\right]$ where $a_{0, j}, j=5,6,7$ are unknowns to be determined.

Let us examine subintervals $\left[x_{i}, x_{i+1}\right], \mathrm{i}=1,2, \ldots, \mathrm{n}-2$. By taking into account the interpolating conditions, we can write the expression, for $S_{i}(x)$ in the following form, see([1-2], [10] and [11]).

$$
\begin{aligned}
& s_{i}(x)=y_{i}+\left(x-x_{0}\right) a_{i, 1}+\frac{\left(x-x_{i}\right)^{2}}{2} y_{i}^{\prime \prime}+\left(x-x_{i}\right)^{3} a_{i, 3}+\frac{\left(x-x_{i}\right)^{4}}{24} y_{i}^{(4)}+\left(x-x_{i}\right)^{5} a_{i, 5}+ \\
& \left(x-x_{i}\right)^{6} a_{i, 6}+\frac{\left(x-x_{i}\right)^{7}}{5040} y_{i}^{(7)}+\left(x-x_{i}\right)^{8} a_{i, 8}+\left(x-x_{i}\right)^{9} a_{i, 9}
\end{aligned}
$$

where $a_{i, j}, i=1(1)(n-1), j=1,3,5,6,8,9$. are unknown values to be determine.

\section{CONVERGENCE ANALYSIS}

In this section, we investigate the convergence analysis of the method for ninth degree spline function which are developed by [2], [4], [10] and [11]. The equation (2) yields the two initial conditions $S^{\prime}\left(x_{0}\right)=y^{\prime}\left(x_{0}\right)$ and $S^{\prime \prime \prime}\left(x_{0}\right)=y^{\prime \prime \prime}\left(x_{0}\right)$, and then we apply the boundary conditions $S^{(r)}\left(x_{i}\right)=y^{(r)}\left(x_{i}\right), r=0,2,4,7$, for $i=0,1, \ldots, n$ in equation (3), to obtain the following: 


$$
\begin{aligned}
a_{0,5}= & \frac{198}{41 h^{5}}\left[y_{1}-y_{0}\right]-\frac{198}{141 h^{4}} y_{0}^{\prime}-\frac{239}{410 h^{2}} y_{0}^{\prime \prime \prime}-\frac{1}{410 h^{3}}\left[91 y_{1}^{\prime \prime}+899 y_{0}^{\prime \prime}\right]+ \\
& \frac{h}{4920}\left[25 y_{1}^{(4)}-469 y_{0}^{(4)}\right]-\frac{h^{2}}{516600}\left[12 y_{1}^{(7)}-35 y_{0}^{(7)}\right], \\
a_{0,6}= & -\frac{192}{41 h^{6}}\left[y_{1}-y_{0}\right]+\frac{192}{41 h^{5}} y_{0}^{\prime}+\frac{617}{1230 h^{3}} y_{0}^{\prime \prime \prime}+\frac{1}{3099600 h^{4}}\left[864360 y_{1}^{\prime \prime}+6393240 y_{0}^{\prime \prime}\right] \\
- & \frac{1}{3099600 h}\left[23100 y_{1}^{(4)}-195720 y_{0}^{(4)}\right]+\frac{h}{3099600}\left[122 y_{1}^{(7)}-595 y_{0}^{(7)}\right], \\
a_{0,8}= & \frac{45}{41 h^{8}}\left[y_{1}-y_{0}\right]-\frac{45}{41 h^{7}} y_{0}^{\prime}-\frac{9}{82 h^{5}} y_{0}^{\prime \prime \prime}-\frac{1}{826560 h^{6}}\left[60480 y_{1}^{\prime \prime \prime}+393129 y_{0}^{\prime \prime \prime}\right] \\
+ & \frac{1}{36400 h^{3}}\left[20 y_{1}^{(5)}+25 y_{0}^{(5)}\right]-\frac{1}{1834560 h}\left[64 y_{1}^{(7)}+161 y_{0}^{(7)}\right], \\
\text { and } & a_{0,9}=\frac{-10}{41 h^{9}}\left[y_{1}-y_{0}\right]+\frac{10}{41 h^{8}} y^{\prime}+\frac{1}{41 h^{6}} y_{0}^{\prime \prime \prime}+\frac{1}{2479680 h^{7}}\left[40320 y_{1}^{\prime \prime \prime}+262080 y_{0}^{\prime \prime \prime}\right] \\
- & \frac{1}{2479680 h^{5}}\left[1680 y_{1}^{(4)}-6720 y_{0}^{(4)}\right]+\frac{1}{2479680 h^{2}}\left[29 y_{1}^{(7)}+35 y_{0}^{(7)}\right] .
\end{aligned}
$$

Substituting these valuesof $a_{0,5}, a_{0,6}, a_{0,8}$ and $a_{0,9}$ in equation (2), we obtain:

$$
\begin{aligned}
a_{1,1}= & \frac{108}{41 h}\left[y_{1}-y_{0}\right]-\frac{67}{41} y_{0}^{\prime}-\frac{13 h^{2}}{205} y_{0}^{\prime \prime \prime}+\frac{h}{410}\left[51 y_{0}^{\prime \prime}-181 y_{0}^{\prime \prime}\right]- \\
& \frac{h^{3}}{4920}\left[5 y_{1}^{(4)}+21 y_{0}^{(4)}\right]+\frac{h^{6}}{4132800}\left[11 y_{1}^{(7)}-15 y_{0}^{(7)}\right],
\end{aligned}
$$

and

$$
\begin{gathered}
6 a_{1,3}=\frac{-1080}{41 h^{3}}\left[y_{1}-y_{0}\right]+\frac{1080}{41 h^{2}} y_{0}^{\prime}+\frac{67}{41} y_{0}^{\prime \prime \prime}+\frac{1}{41 h}\left[154 y_{1}^{\prime \prime}+386 y_{0}^{\prime \prime}\right]+ \\
\frac{h}{246}\left[23 y_{1}^{(4)}+31 y_{0}^{(4)}\right]-\frac{h^{4}}{103320}\left[13 y_{1}^{(7)}-14 y_{0}^{(7)}\right] .
\end{gathered}
$$

We shall find the coefficients of $S_{i}(x)$ for $i=1,2,3, \ldots ., n-1$. From equation (3) we have:

$$
\begin{aligned}
a_{i, 5}= & \frac{198}{41 h^{5}}\left[y_{i+1}-y_{i}\right]-\frac{198}{41 h^{4}} a_{i, 1}-\frac{717}{205 h^{2}} a_{i, 3}-\frac{1}{516600 h^{3}}\left[114660 y_{i+1}^{\prime \prime}+113274 y_{i}^{\prime \prime}\right] \\
+ & \frac{1}{516600 h}\left[2625 y_{i+1}^{(4)}-49245 y_{i}^{(4)}\right]-\frac{h^{2}}{516600}\left[12 y_{i+1}^{(7)}-35 y_{i}^{(7)}\right], \\
a_{i, 6}= & \frac{-192}{141 h^{6}}\left[y_{i+1}-y_{i}\right]+\frac{192}{41 h^{5}} a_{i, 1}+\frac{617}{205 h^{3}} a_{i, 3}+\frac{1}{3099600 h^{4}}\left[864360 y_{i+1}^{\prime \prime}+6393240 y_{i}^{\prime \prime}\right] \\
& -\frac{1}{3099600 h^{2}}\left[23100 y_{i+1}^{(4)}-195720 y_{i}^{(4)}\right]+\frac{1}{3099600}\left[122 y_{i+1}^{(7)}-595 y_{i}^{(7)}\right], \\
a_{i, 8}= & \frac{45}{41 h^{8}}\left[y_{i+1}-y_{i}\right]-\frac{45}{41 h^{7}} a_{i, 1}-\frac{27}{41 h^{5}} a_{i, 3}-\frac{1}{826560 h^{6}}\left[60480 y_{i+1}^{\prime \prime}+393120 y_{i}^{\prime \prime}\right]+ \\
& \frac{1}{826560 h^{3}}\left[2520 y_{i+1}^{(4)} 10080 y_{i}^{(4)}\right]-\frac{1}{826560 h}\left[23 y_{i+1}^{(7)}+73 y_{i}^{(7)}\right],
\end{aligned}
$$

and

$$
\begin{aligned}
& a_{i, 9}=\frac{-10}{41 h^{9}}\left[y_{i+1}-y_{i}\right]+\frac{10}{41 h^{8}} a_{i, 1}+\frac{6}{41 h^{6}} a_{i, 3}+\frac{1}{2479680 h^{7}}\left[60480 y_{i+1}^{\prime \prime}+262080 y_{i}^{\prime \prime}\right] \\
& -\frac{1}{2479680 h^{4}}\left[1680 y_{i+1}^{(4)}-6720 y_{i}^{(4)}\right]+\frac{1}{2479680 h^{2}}\left[29 y_{i+1}^{(7)}+35 y_{i}^{(7)}\right] .
\end{aligned}
$$


Substituting the values of $a_{i, 5}, a_{i, 6}, a_{i, 8}$ and $a_{i, 9}$ in the equation (3), we obtain the following relation,for $i=1,2, \ldots, n-1$.

$$
\begin{aligned}
a_{i+1,1} & =\frac{108}{41 h}\left[y_{i+1}-y_{i}\right]-\frac{108}{41} a_{i}-78 \frac{13 h^{2}}{205} a_{i, 3}+\frac{h}{410}\left[51 y_{i+1}^{\prime \prime}-181 y_{i}^{\prime \prime}\right]- \\
& \frac{h^{3}}{4920}\left[5 y_{i+1}^{(4)}+21 y_{i}^{(4)}\right]+\frac{h^{6}}{4132800}\left[11 y_{i+1}^{(7)}-15 y_{i}^{(7)}\right],
\end{aligned}
$$

and

$$
\begin{gathered}
6 a_{i+1,3}=\frac{-1080}{41 h^{3}}\left[y_{i+1}-y_{i}\right]+\frac{1080}{41 h^{2}} a_{i, 1}+\frac{402}{41} a_{i, 3}+\frac{1}{41 h}\left[154 y_{i+1}^{\prime \prime}+386 y_{i}^{\prime \prime}\right]+ \\
\frac{h}{246}\left[23 y_{i+1}^{(4)}+31 y_{i}^{(4)}\right]-\frac{h^{4}}{103320}\left[13 y_{i+1}^{(7)}-14 y_{i}^{(7)}\right] .
\end{gathered}
$$

Now the coefficient matrixof the above system of equations can be found, in the unknowns $a_{i, 1}, a_{i, 3}, a_{i+1,1}$ and $a_{i+1,3}$ $, i=1,2, \ldots, n-1$ which is a non-singular matrix and hence all the coefficients are determined uniquely.

Theorem 1: Let $y(x)$ be the exact solution of the system (1) and we assumingthat $y_{i}, i=1,2, \ldots, n-1$, be the numerical solution of (1), and $S(x)$ be a unique ninth spline function whichis a solution of the problem (2). Thenfor $x \in\left[x_{0}, x_{1}\right]$, we have:

$$
\left\|S_{0}^{(9-r)}(x)-y^{(9-r)}(x)\right\| \leq\left\{\begin{array}{lc}
A h^{r} w_{9}(f ; h), r=9, & \frac{49}{47232} h^{r} w_{9}(f ; h), r=8 \\
B h^{r} w_{9}(f ; h), r=7, & \frac{1823}{68880} h^{r} w_{9}(f ; h), r=6 \\
\frac{125}{1148} h^{r} w_{9}(f ; h), r=5, & \frac{893}{2296} h^{r} w_{9}(f ; h), r=4 \\
\frac{675}{574} h^{r} w_{9}(f ; h), r=3, & \frac{229}{82} h^{r} w_{9}(f ; h), r=2 \\
\frac{182}{41} h^{r} w_{9}(f ; h), r=1, & \frac{135}{41} h^{r} w_{9}(f ; h), r=0 .
\end{array}\right.
$$

where $A=\frac{323324628002651}{57640752303423423488}, B=\frac{1551065516263567}{9223372036854775808}$

And where $W_{9}(f ; h)$ denotes the modules of continuity of $y^{(9)}$.

Proof:Let $x \in\left[x_{0}, x_{1}\right]$, from equation (2.1) and by using Taylor's expansion formula we get

$S_{0_{i}}^{(9)}(x)-y^{(9)}(x)=362880 a_{0,9}-y_{i}^{(9)}(x) .(4)$

Using (2) and $a_{0,9}$, we obtain

$\left|S_{0}^{(9)}(x)-y^{(9)}(x)\right|=\left|362880 a_{0,9}-y^{(9)}(x)\right| \leq \frac{135}{41} w_{9}(f ; h)$.(5)

By taking the eight derivatives and subtracting the function, using Taylor's series expansion on $y^{(9)}(x)$ about $x=x_{1}$, we $\quad \operatorname{get}\left|S_{0}^{(8)}(x)-y^{(8)}(x)\right|=\left|40320 a_{0,8}+362880\left(x-x_{1}\right) a_{0,9}-y^{(8)}\left(x_{0}\right)+\left(x-x_{0}\right) y^{(9)}\left(\alpha_{1}\right)\right|$

$\leq \frac{182}{41} h w_{9}(f ; h)$ where $x_{0}<\alpha_{1}<x_{1}$.(6)

Also, from (2) and bytaking the seventh derivatives, we get

$S_{0}^{(7)}(x)-y^{(7)}(x)=y_{0}^{(7)}+40320 a_{0,8}+181440 h^{2} a_{0,9}-y^{(7)}(x)$,

hence

$\left|S_{0}^{(7)}(x)-y^{(7)}(x)\right| \leq \frac{229}{82} h^{2} w_{9}(f ; h)$ 
and by taking the sixth derivatives and fromthe Taylor's series expansion on $y^{(6)}(x)$ about $x=x_{1}$, we get: $\left|S_{0}^{(6)}(x)-y^{(6)}(x)\right| \leq \frac{675}{574} h^{3}\left|y^{(9)}\left(\beta_{1}\right)-y^{(9)}\left(\beta_{2}\right)\right| \leq \frac{675}{574} h^{3} w_{9}(f ; h)$ where $x_{0}<\beta_{1}, \beta_{2}<x_{1} \quad$ (8)

From equations (2) and substituting the coefficients, we get: $\left|S_{0}^{(5)}(x)-y^{(5)}(x)\right| \leq \frac{893}{2296} h^{4} w_{9}(f ; h)(9)$

By continuing this process, we have

$\left|S_{0}^{(4)}(x)-y^{(4)}(x)\right| \leq \frac{125}{1148} h^{5} w_{9}(f ; h),\left|S_{0}^{(3)}(x)-y^{(3)}(x)\right| \leq \frac{1823}{68880} h^{6} w_{9}(f ; h)$,

$\left|S_{0}^{\prime \prime}(x)-y^{\prime \prime}(x)\right| \leq \frac{323324628002651}{57640752303423423488} h^{7} w_{9}(f ; h)$

$\left|S_{0}^{\prime}(x)-y^{\prime}(x)\right| \leq \frac{49}{47232} h^{8} w_{9}(f ; h)$

and $\left|S_{0}(x)-y(x)\right| \leq \frac{1551065516263567}{9223372036854775808} h^{9} w_{9}(f ; h)$.

Lemma 1: Let $y(x)$ be the exact solution of the system of (1.1). Assume that $y_{i}, i=1,2, \ldots, n-1$, be the numerical solutions of (1), then $\left|e_{i, 1}\right| \leq c_{i} h^{8} w_{9}(f ; h)$ where

$e_{i, 1}=a_{i, 1}-y_{i}^{\prime}$,

and $\boldsymbol{C}_{\boldsymbol{i}}$ depend on the numbers of intervals.

Proof: For $y(x) \in C^{9}[0,1]$ then using Taylor's expansion formula, we have:

$y(x)=y\left(x_{i}\right)+\left(x-x_{i}\right) y^{\prime}\left(x_{i}\right)+\frac{\left(x-x_{i}\right)^{2}}{2} y^{\prime \prime}\left(x_{i}\right)+\ldots .+\frac{\left(x-x_{i}\right)^{9}}{5040} y^{(9)}\left(\theta_{i}\right)$,

where $x_{i}<\alpha_{i}<x_{i+1}$, and similar expressions for the derivatives of $\mathrm{y}(\mathrm{x})$ can be used.

Now if $\mathrm{i}=1$ then from equation of $a_{1,1}$ and by using (10) we obtain

$$
\begin{aligned}
e_{1,1}=a_{1,1}- & y_{1}^{\prime}=\frac{h^{8}}{137760} y^{(9)}\left(\alpha_{1}\right)-\frac{h^{8}}{40320} y^{(9}\left(\alpha_{2}\right)+ \\
& \frac{17 h^{8}}{688800} y^{(9)}\left(\alpha_{3}\right)-\frac{h^{8}}{118080} y^{(9)}\left(\alpha_{5}\right)+\frac{11 h^{8}}{8265600} y^{(9)}\left(\alpha_{6}\right)
\end{aligned}
$$

where $x_{0}<\alpha_{1}, \alpha_{2}, \alpha_{3}, \alpha_{4}, \alpha_{5}, \alpha_{6}<x_{1}$.

fromequation (11) we get :

$\left|e_{1,1}\right| \leq \frac{h^{8}}{330624} w_{9}(f ; h) \rightarrow\left|e_{1,1}\right| \leq h^{8} c_{1} w_{9}(f ; h)$

where $c_{1}=\frac{1}{330624}$ Also, if $\mathrm{i}=2$, then from the expression of $a_{2,1}$ and by using (10) we obtain

$$
\left|e_{2,1}\right| \leq c_{2} h^{8} w_{9}(f ; h)
$$

Continuing the same process, the inequality $\left|e_{i, 1}\right| \leq c_{i} h^{8} w_{9}(f ; h)$ for $\mathrm{i}=1, \ldots, \mathrm{n}-1$ holds.

Lemma 2: Let $y(x)$ be the exact solution of the system of (1.1). Assume that $y_{i}, i=1,2, \ldots, n-1$, be the numerical solutions of (1), then $\left|e_{i, 3}\right| \leq c_{i}^{\prime} h^{6} w_{9}(f ; h)$ for $\mathrm{i}=1, \ldots, \mathrm{n}-1$

where $e_{i, 3}=6 a_{i, 3}-y_{i}^{\prime \prime \prime}$

and $\boldsymbol{C}_{i}^{\prime}$ depend on the numbers of intervals. 
Proof:Let $y(x) \in C^{9}[0,1]$ and by using the Taylor's expansion formula and similar expressions for the derivatives of $\mathrm{y}$ ( $\mathrm{x}$ ), we have, if $\mathrm{i}=1$ then from the expressions $a_{1,3}$ and from equation (12), we obtain

$$
\begin{gathered}
e_{1,3}=6 a_{1,3}-y_{1}^{\prime \prime \prime}=-\frac{h^{6}}{13776} y^{(9)}\left(\alpha_{1}\right)+\frac{11 h^{6}}{14760} y^{(9}\left(\alpha_{2}\right)- \\
\frac{h^{6}}{720} y^{(9)}\left(\alpha_{3}\right)+\frac{23 h^{6}}{29520} y^{(9)}\left(\alpha_{5}\right)-\frac{13 h^{6}}{206640} y^{(9)}\left(\alpha_{6}\right)
\end{gathered}
$$

where $x_{0}<\alpha_{1}, \alpha_{2}, \alpha_{3}, \alpha_{4}, \alpha_{5}, \alpha_{6}<x_{1}$.

and thus, we get: $\left|e_{1,3}\right| \leq \frac{h^{6}}{656} w_{9}(f ; h)$ and $c_{1}^{\prime}=\frac{1}{656}$

Also, if $\mathrm{i}=2$ then from $a_{i, 3}$ andequation (12), we get $\left|e_{2,3}\right| \leq c_{2}^{\prime} h^{6} w_{9}(f ; h)$

Similarly the inequality $\left|e_{i, 3}\right| \leq c_{i}^{\prime} h^{7} \boldsymbol{w}_{9}(f ; h)$ for $\mathrm{i}=1, \ldots, \mathrm{n}-1$ holds.

Theorem 2: Let $S(x)$ be a unique spline function of ninth degreeand $y(x) \in C^{9}[0,1]$ be the solution of (2). Then for $x \in\left[x_{i}, x_{i+1}\right] ; \mathrm{i}=1,2, \ldots, \mathrm{n}-1$, the following error bounds are holds:

$$
\begin{aligned}
& \left\|S^{(r)}(x)-y^{(r)}(x)\right\| \leq\left\{\begin{array}{l}
\frac{h^{9}}{a}\left(a c_{i}+b c_{i}^{\prime}+c\right) w_{9}(f ; h), \quad r=0, \\
\frac{h^{8}}{47232}\left(47232 c_{i}+23616 c_{i}^{\prime}+49\right) w_{9}(f ; h), r=1, \\
\left(h^{7} c_{i}^{\prime}+\frac{3233246283002651}{576460752303423488}\right) w_{9}(f ; h), r=2, \\
\frac{i}{68880} h^{6}\left(68880 c_{i}+1823\right) w_{9}(f ; h), r=3, \\
\frac{893 i}{2296} h^{5} w_{9}(f ; h), r=4, \frac{125 i}{1148} h^{4} w_{9}(f ; h), r=5, \frac{675 i}{574} h^{3} w_{9}(f ; h), r=6, \\
\frac{229 i}{82} h^{2} w_{9}(f ; h), r=7, \frac{182 i}{41} h w_{9}(f ; h), r=8, \frac{135 i}{41} w_{9}(f ; h), r=9 .
\end{array}\right. \\
& a=3689348814741903232, b=614891469126816900, c=6204262065054268,
\end{aligned}
$$

Proof: Let $x \in\left[x_{i}, x_{i+1}\right]$ where $\mathrm{i}=1,2, \ldots, \mathrm{n}-1$, then from equation (3) and using Taylor's expansion formula we get $S_{i}^{(9)}(x)-y^{(9)}(x)=362880 a_{i, 9}-y^{(9)}(x)(14)$

Using (2) and $a_{0,9}$, we obtain

$$
\left|S_{i}^{(9)}(x)-y^{(9)}(x)\right|=\left|362880 a_{i, 9}-y^{(9)}(x)\right| \leq \frac{135 i}{41} w_{9}(f ; h)(15)
$$

From (2) and by taking the eight derivatives and subtracting the function $y^{(8)}(x)$, andusing Taylor's series expansion on $y^{(8)}(x)$ about $x=x_{i}$, we get

$$
\left|S_{i}^{(8)}(x)-y^{(8)}(x)\right| \leq \frac{182 i}{41} h w_{9}(f ; h) \text { where } x_{i}<\alpha_{i, 1}<x_{i} .
$$

Also, from (3) and taking the seventh derivatives, we get .

$$
\left|S_{i}^{(7)}(x)-y^{(7)}(x)\right| \leq \frac{229 i}{82} h^{2} w_{9}(f ; h) \quad \text { where } x_{i}<\alpha_{i, 2}<x_{i} \text {. }
$$

And by taking the sixth derivatives with using Taylor's series expansion on $y^{(6)}(x)$ about $x=x_{i}$, we get 
$\left|S_{i}^{(6)}(x)-y^{(6)}(x)\right| \leq \frac{675 i}{574} h^{3}\left|y^{(9)}\left(\alpha_{1}\right)-y^{(9)}\left(\alpha_{2}\right)\right| \leq \frac{675 i}{574} h^{3} w_{9}(f ; h)$

where $x_{i}<\alpha_{i, 3}, \alpha_{i, 4}<x_{i+1}$, and also

$\left|S_{i}^{(5)}(x)-y^{(5)}(x)\right| \leq \frac{125 i}{1148} h^{4} w_{9}(f ; h)$ where $x_{i}<\alpha_{i, 3}, \alpha_{i, 4}, \alpha_{i, 5}<x_{i+1}$

by the same process way we can find that: $\quad\left|S_{i}^{(4)}(x)-y^{(4)}(x)\right| \leq \frac{893 i}{2296} h^{5} w_{9}(f ; h)$,

$\left|S_{i}^{\prime \prime \prime}(x)-y^{\prime \prime \prime}\right| \leq \frac{i h^{6}}{68880}\left(68880 c_{i}^{\prime}+1823\right) w_{9}(f ; h)$

Now using lemma (4) and (5), we obtain

$$
\begin{aligned}
\left|S_{i}^{\prime \prime}(x)-y^{\prime \prime}\right| & \leq h^{7} i c_{i}^{\prime} w_{9}(f ; h)+\frac{3233246283002651 i}{576460752303423488} h^{8} w_{9}(f ; h) \\
& \leq\left(h^{7} i c_{i}^{\prime}+\frac{3233246283002651 i}{576460752303423488}\right) w_{9}(f ; h)
\end{aligned}
$$

$\left|S_{i}^{\prime}(x)-y^{\prime}\right| \leq\left|a_{i, 1}-y_{i}^{\prime}\right|+\frac{h^{2}}{2}\left|6 a_{i, 3}-y_{i}^{\prime \prime}\right|+\left|5 h^{4} a_{i, 5}+6 h^{5} a_{i, 6}+8 h^{7} a_{i, 8}+9 h^{8} a_{i, 9}\right|$

$$
\leq \frac{h^{8}}{47232}\left(47232 c_{i}+23616 c_{i}^{\prime}+49\right) w_{9}(f ; h)
$$

and

$$
\begin{aligned}
& \left|S_{i}(x)-y(x)\right| \leq h^{9}\left(a_{i, 1}-y_{i}^{\prime}\right) w_{9}(f ; h)+\frac{h^{9}}{6}\left(6 a_{i, 3}-y_{i}^{\prime \prime \prime}\right) w_{9}(f ; h)+ \\
& \frac{6204262065054268 h^{9}}{3689348814741903232} w_{9}(f ; h) \leq \frac{h^{9}}{a}\left(a c_{i}+b c_{i}^{\prime}+c\right) w_{9}(f ; h)
\end{aligned}
$$

where

$a=3689348814741903232, b=614891469126816900, c=6204262065054268$.

This proves Theorem 2 for $x \in\left[x_{i}, x_{i+1}\right], i=1,2, \ldots, n-1$

\section{NUMERICAL ILLUSTRATION}

In this section, the nonlinear differential system of Magnetohidrodynamics free convection flow is presented and this problem is referred to[8] and [9]. The problems are tested to the efficiency of the development solutions, and to demonstrate its convergence computationally. The problems have been solved by using our method with different values of step size h; it's tabulated in Tables 1 . These show that our results are more accurate.

Problem 1:[8] Consider the system

$$
\begin{aligned}
& \quad f^{\prime \prime \prime}+f f^{\prime \prime}-\left(f^{\prime}\right)^{2}+g_{s} \theta+g_{c} \varphi-M\left(f^{\prime}\right)^{2}=0 \\
& \theta^{\prime \prime}-r \operatorname{Pr} f^{\prime} \theta+\operatorname{Pr} f \theta^{\prime}+\operatorname{Pr} D f \varphi^{\prime \prime}=0 \\
& \varphi^{\prime \prime}-r S c f^{\prime} \varphi+S c f \varphi^{\prime}+\operatorname{Sc} \operatorname{Sr} \theta^{\prime \prime}=0
\end{aligned}
$$

Where $f=f_{w}, f^{\prime}=1, \theta^{\prime}=1, \varphi^{\prime}=1$, at $\eta=0$,

$f^{\prime}=0, \theta=0, \varphi=0$, at $\eta \rightarrow \infty$,

The following notations will indicate in the tables:

TOL

Tolerance

FS

Total failure steps 
AMAXE Absolute of the maximum errorwith respect to derivatives

and TIME $(\mathrm{ms}) \quad$ The execution time taken in microseconds.

The absolute of maximum error with respect to derivatives defined as [4]:

$$
\begin{aligned}
& \operatorname{AMAXE}_{(\mathrm{f})}^{(\mathrm{i})}=\max _{1 \leq i \leq n}\left\|e_{i}^{(j)}\right\|=\max _{1 \leq i \leq n}\left\|s^{(j)}\left(x_{i}\right)-f^{(j)}\left(x_{i}\right)\right\| \operatorname{AMAXE}_{(\varphi)}^{(\mathrm{i})}=\max _{1 \leq i \leq n}\left\|e_{i}^{(j)}\right\|=\max _{1 \leq i \leq n}\left\|s^{(j)}\left(x_{i}\right)-\varphi^{(j)}\left(x_{i}\right)\right\| \\
& \text { and } \mathrm{AMAXE}_{\theta)}^{(\mathrm{i})}=\max _{1 \leq i \leq n}\left\|e_{i}^{(j)}\right\|=\max _{1 \leq i \leq n}\left\|s^{(j)}\left(x_{i}\right)-\theta^{(j)}\left(x_{i}\right)\right\|
\end{aligned}
$$

Where $j$ be the order of derivatives on whole intervals and $f\left(x_{i}\right), \varphi\left(x_{i}\right)$ and $\theta\left(x_{i}\right)$ are the approximate solution of system (20),we can seefrom Taylors expansion that $S^{\prime}\left(x_{0}\right)=f^{\prime}\left(x_{0}\right)$ and $S^{\prime \prime \prime}\left(x_{0}\right)=f^{\prime \prime \prime}\left(x_{0}\right)$, $S^{\prime}\left(x_{0}\right)=\varphi^{\prime}\left(x_{0}\right)$ and $S^{\prime \prime \prime}\left(x_{0}\right)=\varphi^{\prime \prime \prime}\left(x_{0}\right)$ and $S^{\prime}\left(x_{0}\right)=\theta^{\prime}\left(x_{0}\right)$ and $S^{\prime \prime \prime}\left(x_{0}\right)=\theta^{\prime \prime \prime}\left(x_{0}\right)$.

\begin{tabular}{|c|c|c|c|c|c|c|}
\hline TOL & FS & $\mathrm{AMAXE}_{(\mathrm{f})}^{(0)}$ & $\mathrm{AMAXE}_{(\mathrm{f})}^{(1)}$ & $\mathrm{AMAXE}_{(\mathrm{f})}^{(2)}$ & $\mathrm{AMAXE}_{(\mathrm{f})}^{(3)}$ & TIME(ms) \\
\hline $10^{-1}$ & 0 & $1.022 \times 10^{-1}$ & $20.397 \times 10^{-1}$ & $16.891 \times 10^{-1}$ & $1.701 \times 10^{-1}$ & 0.080082 \\
\hline $10^{-2}$ & 0 & $1 \times 10^{-3}$ & $2.378 \times 10^{-1}$ & $16.53 \times 10^{-2}$ & $17 \times 10^{-3}$ & 0.082031 \\
\hline $10^{-3}$ & 0 & $1 \times 10^{-3}$ & $2.41 \times 10^{-3}$ & $16.5 \times 10^{-3}$ & $17 \times 10^{-4}$ & 0.100690 \\
\hline TOL & FS & $\mathrm{AMAXE}_{(\mathrm{f})}^{(4)}$ & $\mathrm{AMAXE}_{(\mathrm{f})}^{(5)}$ & $\mathrm{AMAXE}_{(\mathrm{f})}^{(6)}$ & $\mathrm{AMAXE}_{(\mathrm{f})}^{(7)}$ & TIME(ms) \\
\hline $10^{-1}$ & 0 & $32.91 \times 10^{-1}$ & $1.4577 \times 10^{-2}$ & $3.099 \times 10^{0}$ & $1.254 \times 10^{-3}$ & 0.080082 \\
\hline $10^{-2}$ & 0 & $3.086 \times 10^{-1}$ & $1.1947 \times 10^{0}$ & $23.309 \times 10^{-1}$ & $2.3481 \times 10^{-4}$ & 0.082031 \\
\hline $10^{-3}$ & 0 & $3.03 \times 10^{-2}$ & $13 \times 10^{-2}$ & $23.31 \times 10^{-1}$ & $2.456 \times 10^{-6}$ & 0.100690 \\
\hline & \multicolumn{6}{|c|}{ Table 1(a): Errors Estimations between $S(\eta)$ and $f(\eta)$. } \\
\hline TOL & FS & $\mathrm{AMAXE}_{(\varphi)}^{(0)}$ & $\mathrm{AMAXE}_{(\varphi)}^{(1)}$ & $\mathrm{AMAXE}_{(\varphi)}^{(2)}$ & $\mathrm{AMAXE}_{(\varphi)}^{(3)}$ & TIME(ms) \\
\hline $10^{-1}$ & 0 & $1.003 \times 10^{-1}$ & $1.539 \times 10^{-1}$ & $34 \times 10^{-4}$ & $4.86 \times 10^{-2}$ & 0.000218 \\
\hline $10^{-2}$ & 0 & $1 \times 10^{-2}$ & $15.3 \times 10^{-3}$ & $13 \times 10^{-4}$ & $49 \times 10^{-4}$ & 0.00020 \\
\hline $10^{-3}$ & 0 & $1 \times 10^{-3}$ & $15 \times 10^{-4}$ & $13.883 \times 10^{-5}$ & $49.138 \times 10^{-5}$ & 0.000214 \\
\hline TOL & FS & $\mathrm{AMAXE}_{(\varphi)}^{(4)}$ & $\mathrm{AMAXE}_{(\varphi)}^{(5)}$ & $\mathrm{AMAXE}_{(\varphi)}^{(6)}$ & $\mathrm{AMAXE}_{(\varphi)}^{(7)}$ & TIME(ms) \\
\hline $10^{-1}$ & 0 & $61.47 \times 10^{-2}$ & $11.667 \times 10^{-2}$ & $3.7679 \times 10^{0}$ & $2.945 \times 10^{-3}$ & 0.000218 \\
\hline $10^{-2}$ & 0 & $5.65 \times 10^{-2}$ & $9.97 \times 10^{-2}$ & $3.768 \times 10^{-1}$ & $7.623 \times 10^{-5}$ & 0.00020 \\
\hline $10^{-3}$ & 0 & $56 \times 10^{-4}$ & $98 \times 10^{-4}$ & $3.77 \times 10^{-2}$ & $6.8301 \times 10^{-7}$ & 0.000214 \\
\hline
\end{tabular}

Table 1:Absolute maximum error for $S(\eta)$ and its derivative with different values of tolerance for the problem1:

Table 1(b): Errors Estimations between $S(\eta)$ and $\varphi(\eta)$.

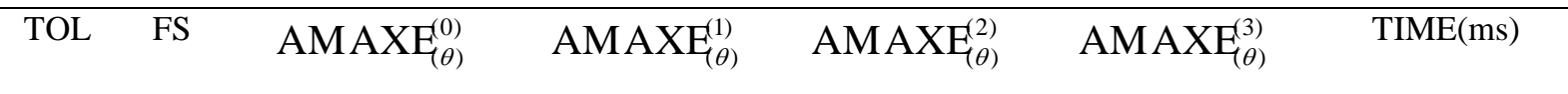




\begin{tabular}{|ccccccc|}
\hline $10^{-1}$ & 0 & $1.003 \times 10^{-1}$ & $8.505 \times 10^{-1}$ & $55 \times 10^{-4}$ & $28.69 \times 10^{-2}$ & 0.000204 \\
$10^{-2}$ & 0 & $1 \times 10^{-2}$ & $8.53 \times 10^{-3}$ & $5 \times 10^{-3}$ & $2.87 \times 10^{-2}$ & 0.000205 \\
$10^{-3}$ & 0 & $1 \times 10^{-3}$ & $8.5 \times 10^{-3}$ & $5.4907 \times 10^{-4}$ & $2.9 \times 10^{-3}$ & 0.000209 \\
\hline TOL & FS & AMAXE $_{(\theta)}^{(4)}$ & AMAXE $_{\theta)}^{(5)}$ & AMAXE $_{(\theta)}^{(6)}$ & AMAXE $_{(\theta)}^{(7)}$ & TIME(ms) \\
\hline $10^{-1}$ & 0 & $24.972 \times 10^{-1}$ & $11.667 \times 10^{-2}$ & $10.8899 \times 10^{0}$ & $0.1248 \times 10^{-4}$ & 0.000204 \\
$10^{-2}$ & 0 & $2.436 \times 10^{-1}$ & $1.005 \times 10^{-1}$ & $1.089 \times 10^{-1}$ & $3.6102 \times 10^{-5}$ & 0.000205 \\
$10^{-3}$ & 0 & $2.43 \times 10^{-2}$ & $9.6 \times 10^{-3}$ & $10.89 \times 10^{-2}$ & $4.456 \times 10^{-7}$ & 0.000209 \\
\hline
\end{tabular}

Table 1(c): Errors Estimations between $S(\eta)$ and $\theta(\eta)$.

\section{CONCLUSION}

The approximate solutions of the nonlinear problems as differential system of Magnetohidrodynamics free convection flow by using ninth spline interpolation show that our method is better in the sense of accuracy and applicability. These have been verified by maximum absolute errors given in the tables it changes with respect to the step size of the tolerance and various problems. Some properties of spline are obtained which are required in proving the uniqueness, existences and convergence analysis of the present method.

\section{RERERENCES}

[1] Ahlberg, J. H.; Nilson, E. N. and Walsh, J. L. , 1967. The theory of splines and their applications, Academic Press, New York, London.

[2]Al Bayati, A. Y.; Saeed, R. K. and Hama-Salh, F. K. , 2009. The Existence, Uniqueness and Error Bounds of Approximation SplinesInterpolation for Solving Second-Order Initial Value Problems, Journal ofMathematics and Statistics ,New York, 5,No.2,123-129.

[3] Eamail, M. N.; Fawzy, Th.; Ahmed, M. and Elmoselhi, H. O., 1994. Deficient spline function approximation to fourth order differential equations, Appl. Math. Modeling, 18, 658-664.

[4] Faraidun, K. H. , 2010. Numerical Solution for Fifth Order Initial Value Problems Using Lacunary Interpolation, Journal of Duhok University, Vol. 13, No. 1, pp.128-134.

[5] Jain M. K.; lyengar, S.R.K. andJian R.K., 2007. Numerical Methods for Scientific and Engineering Computation, fifth edition, new Age International(P) Ltd.

[6] J. Hietarina, 1987. A Search for Bilinear Equations Passing Hirota's Three-Solition Condition, II mKdV-Type Bilinear Equations", Journal of Mathematical Physics, Vol. 28, No. 9, pp. 2094-2101.

[7] Lambert, J.D., 1991. Numerical Methods for Ordinary Differential Systems. ChichesterWiley.

[8] M. U. Ahammad, Md. ShirazulHoqueMollah, 2012. Numerical study of MHD free convection flow and mass transfer over a stretching sheet considering Dufour \& Sort effects in the presence of Magnatic Field, Inter. J. Eng. \& Tech. , IJET-IJENS, Vol:11 No: 5.

[9] O.K. Koriko, 2010. Approximate Solutions of a Higher Order MHD Flow of a Uniformly Stretched Vertical Permeable Surface in the Presence of Heat Generation/Absorption which Resulted from a Quadratic Reaction, Journal of Mathematics and Statistics 6 (2):96-99, ISSN 1549-3644.

[10] Rana, S. S. and Dubey, Y. P., 1999.Best error bounds for deficient quartic spline interpolation, Indian J. Pure Appl. Math., 30 (4), 385-393.

[11] Rostam K. Saeed, Faraidun K. Hamasalh, Gulnar W. Sadiq, 2012.Convergence of Ninth Spline Function to the Solution of a System of Initial Value Problems, World Applied Siences Journal, 16 (10),: 1360-1367.

[12] W. Herman and A. Nuseir, 1997, Symbolic Methods to Construct Exact Solution of Nonlinear Partial Differential Equations, Mathematics and Computers in Simulation, Vol. 43, pp.13-27. 